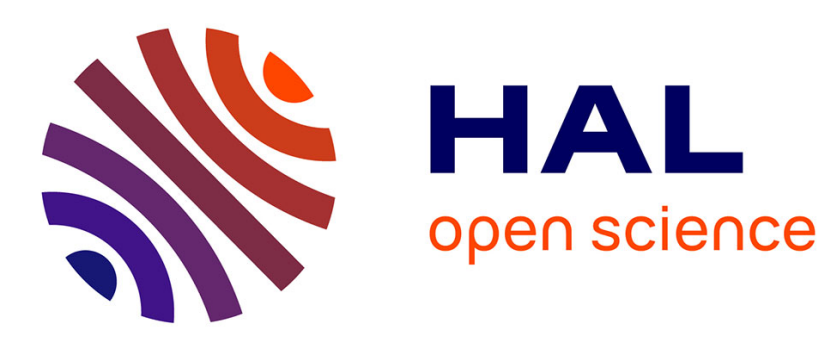

\title{
Multiresolution Analysis for Irregular Meshes with Appearance Attributes
}

\author{
Roy Michael, Sebti Foufou, Frederic Truchetet
}

\section{To cite this version:}

Roy Michael, Sebti Foufou, Frederic Truchetet. Multiresolution Analysis for Irregular Meshes with Appearance Attributes. International Conference on Computer Vision and Graphics, Sep 2004, Varsovie, Poland. pp.80-86. hal-01943976

\section{HAL Id: hal-01943976 https://hal.science/hal-01943976}

Submitted on 4 Dec 2018

HAL is a multi-disciplinary open access archive for the deposit and dissemination of scientific research documents, whether they are published or not. The documents may come from teaching and research institutions in France or abroad, or from public or private research centers.
L'archive ouverte pluridisciplinaire HAL, est destinée au dépôt et à la diffusion de documents scientifiques de niveau recherche, publiés ou non, émanant des établissements d'enseignement et de recherche français ou étrangers, des laboratoires publics ou privés. 


\title{
MULTIRESOLUTION ANALYSIS FOR IRREGULAR MESHES WITH APPEARANCE ATTRIBUTES
}

\author{
Michaël Roy, Sebti Foufou, and Frédéric Truchetet \\ LE2I, CNRS UMR 5158 - Université de Bourgogne \\ 12 rue de la fonderie - 71200 Le Creusot - France
}

\begin{abstract}
We present a new multiresolution analysis framework based on the lifting scheme for irregular meshes with attributes. We introduce a surface prediction operator to compute the detail coefficients for the geometry and the attributes of the model. Attribute analysis gives appearance information to complete the geometrical analysis of the model.We present an application to adaptive visualization and some experimental results to show the efficiency of our framework.
\end{abstract}

Keywords: multiresolution analysis, irregular meshes, appearance attributes, adaptive visualization

\section{INTRODUCTION}

3D scanners usually produce huge data sets containing geometrical and appearance attributes. Geometrical attributes describe shape and dimensions of the object and include data relative to a point set on the object surface. Appearance attributes describe object surface properties such as colors, texture coordinates, etc.

Multiresolution analysis is an efficient framework to represent a data set at different levels of detail, and to provide frequency information. It gives rise to many applications such as filtering, denoising, compression, editing, etc. Many papers present the multiresolution analysis and the wavelet transform in computer graphics domain. Here we refer to Stollnitz et al., 1996 as an introduction.

The goal of this work is to build a multiresolution mesh analysis managing both geometric and appearance attributes. Attribute management is very important especially for terrain models where the attributes are linked to the nature of the terrain. In some cases, attributes are more important than the terrain itself. The main contributions of this work are the use of the lifting 
scheme for the multiresolution analysis of irregular meshes with attributes, and the application of the proposed multiresolution framework to detail coefficient dependent visualization of meshes.

In section 2, we review multiresolution mesh analysis schemes proposed in the literature. In section 3, we detail the proposed multiresolution analysis framework. Section 4 presents some experimental results and the application of the proposed method to detail-dependent visualization. Conclusion and ideas for future extensions are given in section 5 .

\section{Related Work}

Lounsbery et al., 1997 made the connection between wavelets and subdivision to define different levels of resolution. This technique makes use of the theory of multiresolution analysis and of the subdivision rules to construct a multiresolution representation for surfaces with subdivision connectivity. Zorin et al., 1997 proposed a combination of subdivision and smoothing algorithms to construct a set of algorithms for interactive multiresolution editing of complex meshes with arbitrary topology. Bonneau, 1998 introduced the concept of multiresolution analysis over non-nested spaces, which are generated by the so-called BLaC-wavelets. This concept was then used to contruct a multiresolution analysis over irregular meshes. Kobbelt et al., 1998 proposed a multiresolution editing tool for irregular meshes using the progressive mesh algorithm (Hoppe, 1996) to build the coarse resolution mesh, and a smoothing operator to estimate the high resolution mesh. Guskov et al., 1999 presented a series of non-uniform signal processing algorithms designed for irregular triangulation, a smoothing algorithm combined with existing hierarchical methods is used to build subdivision, pyramid, and wavelet algorithms for meshes with irregular connectivity. Recently, Valette and Prost, 2004 presented a new wavelet-based multiresolution analysis of irregular surface meshes using a new irregular subdivision scheme. The method is a fine-to-coarse decomposition, and use a complex simplification algorithm in order to define surface patches suitable for their irregular subdivision scheme.

\section{MULTIRESOLUTION ANALYSIS}

Multiresolution analysis provides a framework that rigorously defines various approximations and fast analysis algorithms. This framework constructs iteratively approximation and detail parts forming successive levels of resolution of the original data set. The details capture the local frequency content of the data set, and are used to exactly reconstruct the original data set.

Classical multiresolution analysis frameworks (such as wavelet transform) cannot be applied to irregularly sampled data sets. Sweldens, 1998 proposed the lifting scheme that allows the multiresolution analysis of irregular samples. 
We propose a multiresolution analysis framework suitable for triangular irregular meshes with appearance attributes. This framework decomposes a mesh in a series of levels of detail, and computes detail coefficients at each level.

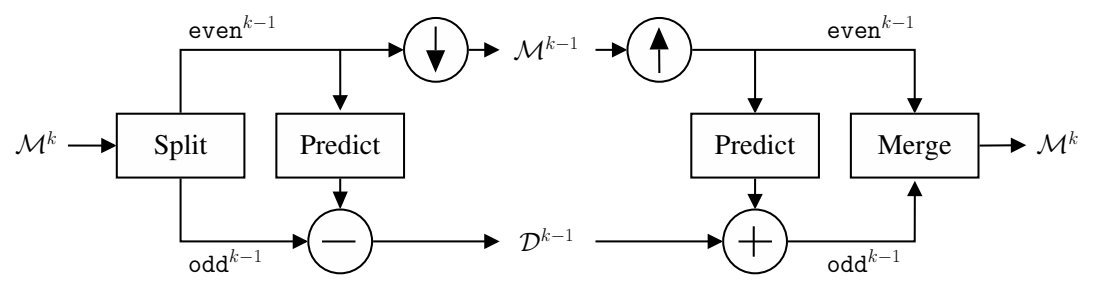

Figure 1. Multiresolution mesh analysis framework.

The multiresolution mesh analysis framework is presented in Fig. 1. The decomposition is represented on the left part of the figure. Starting from a fine mesh $\mathcal{M}^{k}$, two groups of vertices (odds and evens) are defined by the split operator. The odd vertices are designated to be removed, and the even vertices remain to create the coarse mesh $\mathcal{M}^{k-1}$ that approximates the inital mesh. The odd vertices are predicted using the predict operator, and then substracted to the original odd vertices to give the detail coefficients $\mathcal{D}^{k-1}$. The last step is the removal of the odd vertices from the initial mesh using a downsampling operator. The reconstruction is shown on the right part of the figure and is simply the inverse scheme. Starting from a coarse mesh $\mathcal{M}^{k-1}$, the odd vertices are re-inserted using an upsampling operator. The odd vertices are predicted using the predict operator, and then exactly reconstructed by adding the detail coefficients $\mathcal{D}^{k-1}$. In the following paragraphs, we detail the different steps required to build the multiresolution mesh analysis framework.

\section{Downsampling and Upsampling}

We employ the Progressive Mesh (PM) framework (Hoppe, 1996) to build downsampling and upsampling operators. In the PM setting an edge collapse provides the atomic downsampling step, and a vertex split becomes the atomic upsampling step. For the downsampling, we use the half-edge collapse operation, which does not introduce a new vertex position but rather subsamples the original mesh. Thus, it enables the contruction of nested hierarchies on unstructured meshes that can facilitate further applications. The downsampling operator removes about $33 \%$ of the vertices per level.

\section{Split and Merge}

The split operator takes a given mesh and selects the even and the odd vertices. The later are designated to be removed using half-edge collapse operations. In order to do global downsampling and upsampling, the odd vertices 
are defined as a set of independent vertices (not directly connected by an edge). Different methods can be used to select the odd vertices, and thus the half-edge collapses to perform. Our algorithm performs an incremental selection by selecting one odd vertex and locking all adjacent vertices (even vertices). The selection ends when no more vertices can be selected. By selecting an odd vertex in order to remove it, we also select the even vertex it will merge with. In other words, we directly select a half-edge collapse. The Quadric Error Metric from Garland and Heckbert, 1997 is used as a criterion for the selection of the odd vertices because it minimizes the length of the details and retains the visual appearance of the simplified mesh. Since the upsampling is completly done with vertex split operations, the merge operator is not required.

\section{Predict}

The predict operator estimates the odd vertices using the even vertices. We propose a prediction operator that uses the local surface geometry. Meshes coming from real world scenes usually contain appearance attributes such as colors, texture coordinates, etc. Also, we consider the vertex position and the normal vector as geometric attributes. Attributes are considered as vectors in Euclidian space defined on each vertex of the mesh. So a vertex is represented as an array composed of $m$ attribute vectors $\left(\mathbf{a}_{\mathbf{1}}, \ldots, \mathbf{a}_{\mathbf{m}}\right)$ where each $\mathbf{a}_{\mathbf{n}}$ is an attribute vector. We define an application $\mathbf{f}_{\mathbf{n}}(v)=\mathbf{a}_{\mathbf{n}}$ that gives the attribute vector $\mathbf{a}_{\mathbf{n}}$ of the attribute $n$ associated with the vertex $v$. Our predicition operator estimates each odd vertex $v_{i}^{k}$ from the mesh $\mathcal{M}^{k}$ as a set of attributes $\mathbf{f}_{\mathbf{n}}\left(v_{i}^{k}\right)$ given by :

$$
\mathbf{f}_{\mathbf{n}}\left(v_{i}^{k}\right)=\sum_{j \in \mathcal{V}_{1}^{k}(i)} w_{i, j}^{k} \cdot \mathbf{f}_{\mathbf{n}}\left(v_{j}^{k}\right) .
$$

$\mathcal{V}_{1}^{k}(i)$ represents the one-ring neighborhood of the vertex $v_{i}^{k}$. The $w_{i, j}$ are weights of the relaxation operator minimizing the curvature energy of an edge $e_{i, j}$ (Meyer et al., 2002) :

$$
w_{i, j}^{k}=\frac{\cot \alpha_{i, j}+\cot \beta_{i, j}}{\sum_{l \in \mathcal{V}_{1}^{k}(i)} \cot \alpha_{i, l}+\cot \beta_{i, l}}
$$

where $\alpha_{i, j}$ and $\beta_{i, j}$ are the angles opposite to the edge $e_{i, j}$. Predicted attributes are relaxed in terms of curvature energy of the analysed surface. This relaxation operator guaranties smooth variation of the attributes. This method also assumes that the attributes are linked to the surface. The $w_{i, j}$ coefficients are computed during the decomposition, and need to be stored to be re-used for the prediction step in the reconstruction. 


\section{EXPERIMENTAL RESULTS}

We present an application of our multiresolution framework to adaptive visualization. Our method uses the PM framework, which has proved its efficiency for view-dependent visualization of meshes (Hoppe, 1997). The PM framework selects the vertices to display using visiblity criteria. We improve this selection using the detail coefficients of the analysis. If the detail coefficient length of a vertex is below a given threshold, the vertex is declared as irrelevant and thus is removed from the mesh.

Figure 2 shows view-dependent visualization of the Buddha and the Earth model. On both Figures 2(a) and 2(b), the viewport used to visualize the models is shown as the yellow pyramid. We see that invisible vertices are removed using frustrum and backface culling. Irrelevant vertices are removed by thresholding the detail coefficients.

Figure 3 shows view and detail dependent visualization of the Buddha model using the viewport represented in Fig. 2(a). The original model, from Stanford University, contains 543.652 vertices and 1.087.716 faces. Figure 3(a) shows the model visualized using only frustrum and backface culling. Figures 3(b) and 3(c) show the Buddha model visualized with our detail dependent technique. Each figure shows the result of different values of the threshold (the higher the threshold, the coarser the resolution). We see that important geometrical features of the model such as the high curvature regions are preserved.

Figure 4(a) shows a model of the earth with color attributes. Figure 4(b) shows the same model simplified using our method. The detail coefficients of the color attributes are thresholded to remove irrelevant vertices. Detail dependent simplification insures the preservation of attribute features (e.g. the coastlines). The advantage of our method is that it allows more advanced visualization by computing the relevance of the vertices using the detail coefficients. Combinations of several attributes can be performed to improve the result of the simplification.

\section{CONCLUSION AND FUTURE WORK}

We have presented a new multiresolution analysis for irregular meshes based on the lifting scheme. Our framework manages attributes such as color, normals, etc. Our method is easy to implement and results show the efficiency of the analysis over the attributes, which allows more complete multiresolution analysis. The next step in this work will focus on feature detection using detail orientation in order to build a semi-automatic denoising algorithms for scanned models. 


\section{References}

Bonneau, G.-P. (1998). Multiresolution analysis on irregular surface meshes. IEEE Transactions on Visualization and Computer Graphics, 4(4):365-378.

Garland, M. and Heckbert, P. (1997). Surface simplification using quadric error metrics. In Proceedings of ACM SIGGRAPH, pages 209-216.

Guskov, I., Sweldens, W., and Schröder, P. (1999). Multiresolution signal processing for meshes. In Proceedings of ACM SIGGRAPH, pages 325-334.

Hoppe, H. (1996). Progressive meshes. In Proceedings of ACM SIGGRAPH, pages 99-108.

Hoppe, H. (1997). View-dependent refinement of progressive meshes. In Proceedings of ACM SIGGRAPH, pages 189-198.

Kobbelt, L., Campagna, S., Vorsatz, J., and Seidel, H.-P. (1998). Interactive multi-resolution modeling on arbitrary meshes. In Proceedings of ACM SIGGRAPH, pages 105-114.

Lounsbery, M., DeRose, T., and Warren, J. (1997). Multiresolution analysis for surfaces of arbitrary topological type. ACM Transactions on Graphics, 16(1):34-73.

Meyer, M., Desbrun, M., Schröder, P., and Barr, A.H. (2002). Discrete differential-geometry operators for triangulated 2-manifolds. In Proceedings of Visualization and Mathematics.

Stollnitz, E. J., DeRose, T. D., and Salesin, D. H. (1996). Wavelets for Computer Graphics: Theory and Applications. Morgan Kaufmann.

Sweldens, W. (1998). The lifting scheme: A construction of second generation wavelets. SIAM Journal on Mathematical Analysis, 29(2):511-546.

Valette, S. and Prost, R. (2004). Wavelet-based multiresolution analysis of irregular surface meshes. IEEE Transactions on Visualization and Computer Graphics, 10(2):113-122.

Zorin, D., Schröder, P., and Sweldens, W. (1997). Interactive multiresolution mesh editing. In Proceedings of ACM SIGGRAPH, pages 259-269.

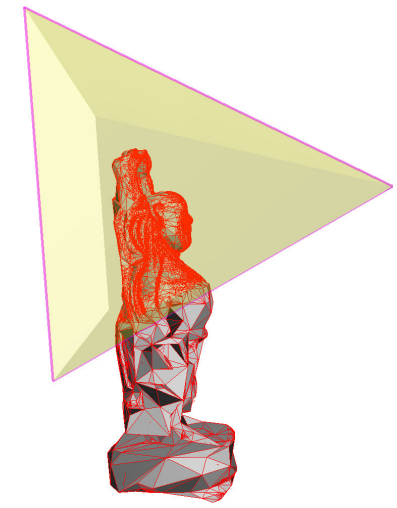

(a)

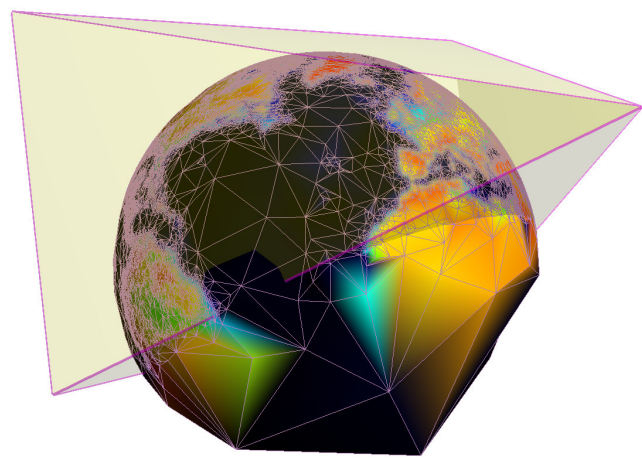

(b)

Figure 2. View and detail dependent visualization of the Buddha model in (a) and the Earth model in (b). Both figures show the viewport used to visualize the models. Wireframe representation is overlaid on each model. 


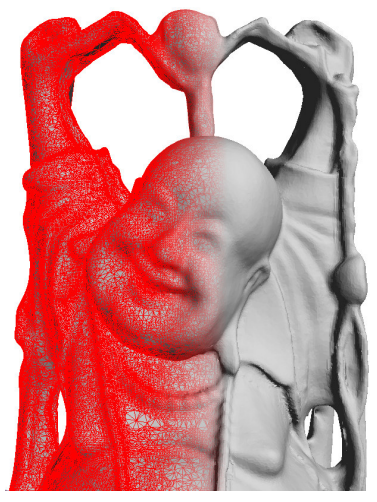

$$
\begin{gathered}
\text { (a) Original model } \\
102.246 \text { vertices } \\
204.522 \text { faces }
\end{gathered}
$$

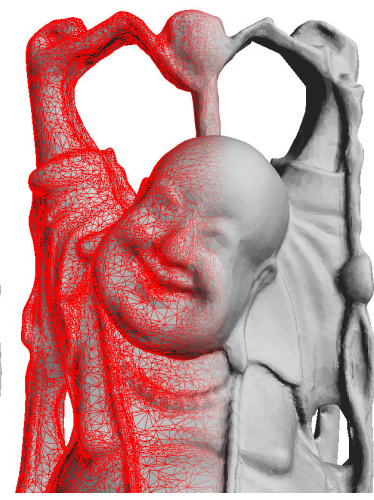

(b) $\tau=0.0001$
31.848 vertices

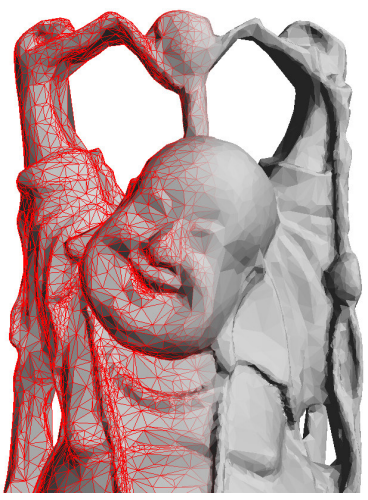

(c) $\tau=0.0004$

9.293 vertices

18.524 faces

Figure 3. Detail dependent visualization of the Buddha model in (a). Figures (b) and (c) show two different values of the detail threshold used to segment the geometric details.

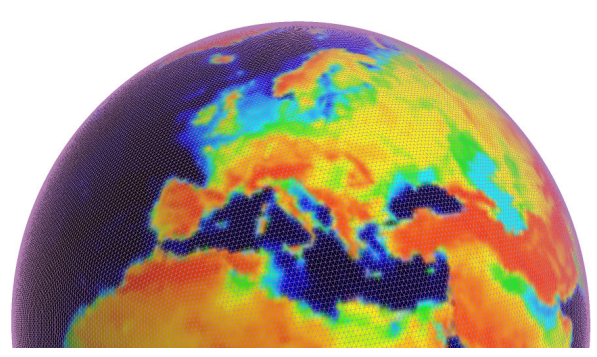

(a) Original model (327,680 faces)

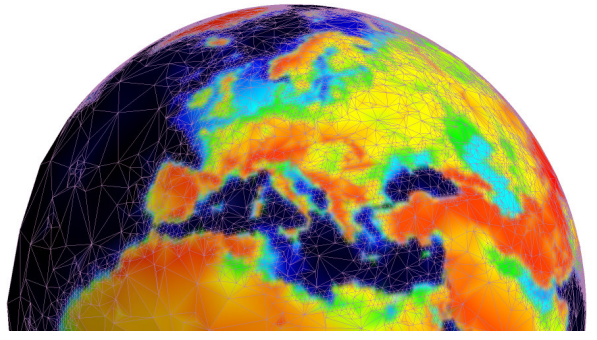

(b) Simplified model (62,632 faces)

Figure 4. Adaptive visualization according to the color detail coefficients. Original model is shown in (a), and simplified model in (b). Relevant vertices are selected by thresholding the color detail coefficients. The model is a sphere with color representing the elevation of the earth from the ETOPO5 data set. 